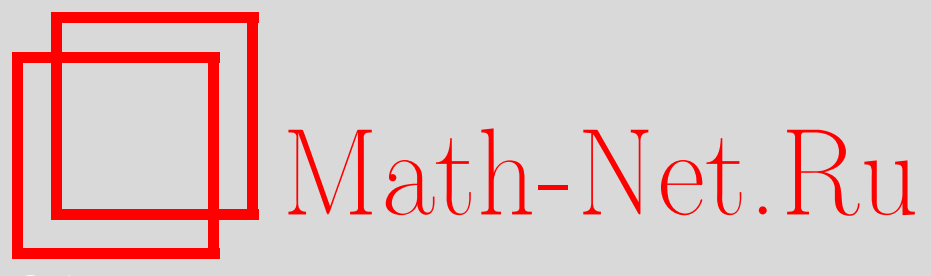

Д. Д. Киселев, О всюду плотной обмотке 2-мерного тора, Матем. сб., 2016, том 207, номер 4, 113-122

DOI: https://doi.org/10.4213/sm8471

Использование Общероссийского математического портала Math-Net.Ru подразумевает, что вы прочитали и согласны с пользовательским соглашением http://www . mathnet.ru/rus/agreement

Параметры загрузки:

IP : 54.210 .77 .194

26 апреля 2023 г., 09:36:54

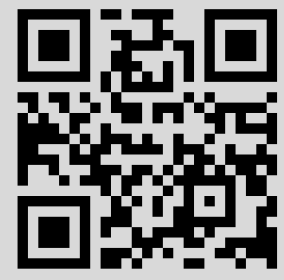




\title{
Д. Д. Киселев
}

\section{О всюду плотной обмотке 2-мерного тора}

\begin{abstract}
В решении некоторого класса задач оптимального управления важную роль играет многочлен специального вида степени $2(n-1)$ с целыми коэффициентами. Линейная независимость набора из $k$ специальных корней этого многочлена над полем $\mathbb{Q}$ влечет существование решения исходной задачи с оптимальным управлением в виде всюду плотной обмотки $k$-мерного клиффордова тора, проходимой за конечное время. В работе показано, что для всех натуральных $n>3$ в качестве $k$ можно выбрать 2 .

Библиография: 6 названий.
\end{abstract}

Ключевые слова: оптимальное управление, всюду плотная обмотка, группа Галуа, линейная независимость.

DOI: $10.4213 / \operatorname{sm} 8471$

\section{§1. Введение}

1.1. Рассмотрим гладкое $2 n$-мерное симплектическое многообразие $M^{2 n}$. Пусть $(2 n-1)$-мерное стратифицированное подмногообразие $S \subset M$ разделяет $M$ на конечное число открытых областей $\Omega_{1}, \ldots, \Omega_{k}: M=\bar{U} \Omega_{i}$. Рассмотрим непрерывный гамильтониан $H(q, p): M \rightarrow \mathbb{R}$, ограничение которого $H_{i}=\left.H\right|_{\Omega_{i}}$ на любое множество $\Omega_{i}$ определяет гладкую функцию, $C^{\infty}$-продолжимую на окрестность множества $\overline{\Omega_{i}}$.

Одной из основных целей теории оптимального управления является построение оптимального синтеза, т.е. фазового портрета экстремалей, проходящих через каждую начальную точку $x_{0}$. Принцип максимума Понтрягина сводит эту проблему к изучению гамильтоновой системы дифференциальных уравнений с разрывной правой частью, причем разрывы достигаются в тех точках $x_{0} \in S$, для которых максимум достигается при нескольких различных значениях управления. В таких точках стандартные теоремы существования и единственности не применимы. Вопросы существования обобщенного решения рассматриваются с помощью вспомогательного дифференциального уравнения с многозначной правой частью. В правой части этого уравнения стоит выпуклое замыкание всех предельных точек фазовых скоростей, полученных при стремлении окрестных точек к точке $x_{0}$. Единственность решения, проходящего через $x_{0}$, принципиально не имеет места. Здесь на первый план выходит проблема описания с помощью функций $H_{i}$ соответствующей интегральной воронки - конуса решений, входящих в точку $x_{0}$ или выходящих из этой точки. Порядок особенности определяется числом кратных скобок Пуассона от функций $H_{i}$, которые обращаются в нуль в точке $x_{0}$ (см. [1]). 
Для одномерного управления, или, что то же самое, для поверхности разрыва коразмерности 1 при особенности первого порядка, в типичной ситуации интегральная воронка состоит из трех экстремалей: две из них лежат по разные стороны от поверхности разрыва, а третья проходит по этой поверхности. При особенности второго порядка ситуация общего положения была найдена и исследована в работах И. Купки [2], М.И. Зеликина и В.Ф. Борисова [3]. Экстремали подходят к точке $x_{0}$ (и отходят от этой точки) за конечное время со счетным числом пересечений поверхности разрыва (chattering-режим).

Особенности второго порядка для многомерного управления в случае, когда областью изменения управления является сфера, были описаны в работах А. А. Милютина [4], М. И. Зеликина и В. Ф. Борисова [3], М. И. Зеликина, Л. В. Локуциевского и Р. Хильдебранда [1]. При подходе к точке $x_{0}$ управление за конечное время совершает счетное число оборотов по некоторой окружности. Соответствующая траектория $x(t)$ попадает в точку $x_{0}$, проходя за конечное время некоторый аналог логарифмической спирали. В ряде случаев возможны комбинации таких решений, лежащих в различных двумерных подпространствах. Феномен заключается в том, что оптимальное управление движется вдоль иррациональной всюду плотной обмотки клиффордова тора, вложенного в сферу. Оптимальная траектория, отвечающая такому управлению, представляет собой обобщенную логарифмическую спираль, натянутую на иррациональную обмотку. В отличие от особенности типа фокуса в обыкновенных дифференциальных уравнениях, движение по этой спирали занимает конечное время, причем оптимальная траектория за конечное время попадает в начало координат. При этом иррациональность обмотки тора достигается за счет линейной независимости над полем $\mathbb{Q}$ корней многочленов с целыми коэффициентами специального вида (см. [1], [5]).

Рассмотрим оптимизационную задачу

$$
J(x)=\int_{0}^{+\infty}\langle x, C x\rangle d t \rightarrow \min
$$

на траекториях управляемой системы

$$
\begin{gathered}
x^{(n)}=u, \quad|u| \leqslant 1, \quad x \in V, \quad u \in U=V, \\
x^{(k)}(0)=x_{k}^{0}, \quad k \leqslant n-1 .
\end{gathered}
$$

Здесь $V$ - конечномерное евклидово пространство достаточно высокой размерности со скалярным произведением $\langle\cdot, \cdot\rangle$, а $C$ - некоторый самосопряженный линейный оператор. Функция $x(t)$ считается абсолютно непрерывной вместе со своими $2 n-1$ производными. Управление $u(t) \in L_{1}(0 ;+\infty)$ - измеримая функция.

В работах [1], [5] было установлено, что в задаче (1.1) существует оптимальное управление, проходящее за конечное время всюду плотную обмотку $k$-мерного тора при $k \leqslant[n / 2]$, если некоторые $k$ корней (из определенным образом выбранного $[n / 2]$-элементного множества корней $A$ ) многочлена $f\left(x^{2}\right)$ 
степени $2(n-1)$, где

$$
x f\left(x^{2}\right)=\operatorname{Im} \prod_{j=1}^{2 n}(i x+j),
$$

линейно независимы над полем $\mathbb{Q}$, а оператор $C$ выбран подходящим образом (см. [5; теоремы 2, 3]). Там же, в [5; лемма 1], показано, что положительный ответ на вопрос о линейной независимости всех корней из множества $A$ можно получить, установив вложение $A_{n-1} \hookrightarrow \operatorname{Gal}_{\mathbb{Q}}(f)$. При $n<16$ данное вложение было установлено (см. $[5 ; \S 3])$.

В настоящей работе мы покажем, что для любого $n>3$ в качестве $k$ можно выбрать 2. Иными словами, в задаче (1.1) при $n>3$ можно, подходящим образом выбрав оператор $C$ согласно [5; теорема 3], построить решение, траектория оптимального управления которого за конечное время проходит всюду плотную обмотку 2-мерного тора.

1.2. Обозначения. Через $\sigma_{k, m}$ мы будет обозначать элементарный симметрический многочлен степени $k$ от $\{j \in \mathbb{N} \mid j \leqslant m\} ; \mathbb{R}_{>0}-$ множество положительных действительных чисел; $\mathbb{R}_{<0}-$ множество отрицательных действительных чисел; $\mathbb{Z}_{\geqslant 0}$ - множество целых неотрицательных чисел.

\section{§2. Основной результат}

2.1. Фиксируем произвольным образом натуральное число $n>3$ и рассмотрим многочлен $f_{n}(x)$, определенный условием

$$
x f_{n}\left(x^{2}\right)=\operatorname{Im} \prod_{j=1}^{2 n}(i x+j) .
$$

Обозначим $F_{n}(x):=f_{n}\left(x^{2}\right)$. Аргумент ${ }^{1}$ множителя $i x+j$ при $x \in \mathbb{R}_{>0}$ принимает все значения из интервала $(0, \pi / 2)$ и возрастает с ростом $x$. Поэтому аргумент произведения $(i x+1) \cdots(i x+2 n)$ пробегает все значения из интервала $(0, \pi n)$ и возрастает с ростом $x$. При $x \in \mathbb{R}_{<0}$ аргумент множителя ${ }^{2}$ принимает все значения из интервала $(-\pi / 2,0)$ и убывает с убыванием $x$. Поэтому аргумент произведения $(i x+1) \cdots(b i x+2 n)$ пробегает все значения из интервала $(-\pi n, 0)$ и убывает с убыванием $x$. Таким образом, многочлен $F_{n}(x)$ сепарабелен, имеет $n-1$ положительных корней и $n-1$ отрицательных корней. В частности, многочлен $f_{n}(x)$ имеет $n-1$ различных положительных действительных корней.

Пусть $n$ четно. Тогда положим $A=\left\{v_{k}\right\}_{k=1}^{[n / 2]} \subset \mathbb{R}_{>0}$, где $v_{k}-$ единственный действительный корень уравнения

$$
\sum_{s=1}^{2 n} \operatorname{arctg} \frac{\sqrt{x}}{s}=(2 k-1) \pi .
$$

\footnotetext{
13десь мы берем значение аргумента как значение соответствующего арктангенса.

${ }^{2}$ И здесь под аргументом мы понимаем значение арктангенса.
} 
Пусть $n$ нечетно. Тогда положим $A=\left\{v_{k}\right\}_{k=1}^{[n / 2]} \subset \mathbb{R}_{>0}$, где $v_{k}$ - единственный действительный корень уравнения

$$
\sum_{s=1}^{2 n} \operatorname{arctg} \frac{\sqrt{x}}{s}=2 k \pi .
$$

Согласно [5; теорема 2] множество $B=\{\sqrt{x} \mid x \in A\}$ и есть "критическое множество" корней многочлена $F_{n}(x)$ : линейная независимость некоторых $k$ корней этого множества над полем $\mathbb{Q}$ влечет существование ${ }^{3}$ решения задачи (1.1), траектории оптимального управления которого проходят за конечное время всюду плотную обмотку $k$-мерного тора ${ }^{4}$.

Выберем произвольным образом 2 элемента множества $B$ и обозначим их $w_{1}, w_{2}$. Если $w_{1}$ и $w_{2}$ линейно зависимы над $\mathbb{Q}$, то и их квадраты также линейно зависимы над $\mathbb{Q}$. Поэтому если мы найдем 2 линейно независимых над $\mathbb{Q}$ элемента множества $A$, то мы найдем 2 линейно независимых над $\mathbb{Q}$ элемента множества $B$. Поэтому в дальнейшем будем работать с множеством $A$.

Продолжая рассуждения, полезно заметить, что многочлен $f_{n}(x)$ имеет вид

$$
f_{n}(x)=(-1)^{n-1} \sigma_{1,2 n} x^{n-1}+(-1)^{n-2} \sigma_{3,2 n} x^{n-2}+\cdots+\sigma_{2 n-1,2 n} .
$$

Лемма 1. Для любого натуралъного $n$ имеем $\sigma_{1,2 n} \mid \sigma_{2 k+1,2 n}$ nри $k \in \mathbb{Z}_{\geqslant 0}$ maкux, чmo $2 k+1<2 n$.

ДокАЗАтеЛьство. Легко видеть, что $\sigma_{1,2 n}=n(2 n+1)$. Также ясно, что $(n, 2 n+1)=1$. Поэтому достаточно показать, что $n \mid \sigma_{2 k+1,2 n}$, а также что $(2 n+1) \mid \sigma_{2 k+1,2 n}$.

Рассмотрим произведение $(i x+1) \cdots(i x+2 n)$. Непосредственная проверка показывает, что мнимая часть данного произведения есть многочлен от $x$, коэффициенты которого равны соответствующим числам $\sigma_{2 k+1,2 n}$, причем коэффициентов вида $\sigma_{2 k, 2 n}$ мнимая часть не содержит. Редуцируем произведение $(i x+1) \cdots(i x+2 n)$ по модулю $2 n+1$. В таком случае мы получим в кольце $(\mathbb{Z} /(2 n+1) \mathbb{Z})[i, x] /\left(i^{2}+1\right)$ равенство

$(i x+1) \cdots(i x+2 n)=(i x+1)(i x-1) \cdots(i x+n)(i x-n)=\left(-x^{2}-1^{2}\right) \cdots\left(-x^{2}-n^{2}\right)$,

т.е. мнимая часть исчезает. Поэтому $(2 n+1) \mid \sigma_{2 k+1,2 n}$.

Редуцируем произведение $(i x+1) \cdots(i x+2 n)$ по модулю $n$. В таком случае мы получим в кольце $(\mathbb{Z} / n \mathbb{Z})[i, x] /\left(i^{2}+1\right)$ равенство

$$
\begin{aligned}
(i x+1) \cdots(i x+2 n) & =-x^{2}(i x+1)(i x-1) \cdots(i x+(n-1))(i x-(n-1)) \\
& =-x^{2}\left(-x^{2}-1^{2}\right) \cdots\left(-x^{2}-(n-1)^{2}\right) .
\end{aligned}
$$

Таким образом, мнимая часть снова исчезает. Итак, $n \mid \sigma_{2 k+1,2 n}$. Лемма доказана.

\footnotetext{
${ }^{3}$ Разумеется, при подходящем выборе оператора $C$, но такой выбор всегда возможен (см. [5; теорема 3]).

${ }^{4}$ При $t>0$ проходится половина обмотки; другая половина проходится при $t<0$, где $t-$ временная переменная.
} 
Лемма 2. Пусть $f(x) \in \mathbb{Q}[x]-$ неприводимый над полем $\mathbb{Q}$ многочлен степени $m \geqslant 2$. Пусть все корни многочлена $f(x)$ вещественны и одного знака. Тогда любые два корня многочлена $f(x)$ линейно независимы над $\mathbb{Q}$.

ДокАзАТЕльство. Фиксируем произвольным образом два корня многочлена $f(x)$ и обозначим их через $v_{1}, v_{2}$. Предположим, что имеется линейная зависимость над $\mathbb{Q}$ вида

$$
\alpha_{1} v_{1}+\alpha_{2} v_{2}=0, \quad \alpha_{1}^{2}+\alpha_{2}^{2} \neq 0 .
$$

В силу неприводимости многочлена $f(x)$ над $\mathbb{Q}$ его группа Галуа действует на корнях транзитивно. Поэтому найдется элемент $g \in \mathrm{Gal}_{\mathbb{Q}}(f)$ такой, что $v_{1}^{g}=v_{2}$. Предположим без ограничения общности, что $\alpha_{2} \neq 0$ в (2.5). Тогда $v_{2}=\lambda v_{1}$, где $\lambda=-\alpha_{1} / \alpha_{2}$. Поэтому $v_{2}^{g}=\lambda v_{2}$, и более общим образом $v_{2}^{g^{s}}=\lambda^{s} v_{2}$. Так как элемент $g$ имеет конечный порядок, то для некоторого натурального $s_{0}$ мы получаем $\lambda^{s_{0}}=1$. Так как $\lambda \in \mathbb{Q}$, то $\lambda \in\{-1,1\}$. Во всех случаях получаем противоречие: при $\lambda=-1$ противоречие с условиями леммы, при $\lambda=1$ противоречие с неприводимостью многочлена $f(x)$ над $\mathbb{Q}$, ибо неприводимость над полем нулевой характеристики влечет сепарабельность. Лемма доказана.

Теперь мы можем исследовать до конца случай четного $n$.

ТеОрема 1. Пусть $n$ - четное натуральное число, большее 3 , а многочлен $f_{n}(x)$ определен согласно (2.4). Пусть $A=\left\{v_{k}\right\}_{k=1}^{n / 2}-$ множество корней многочлена $f_{n}(x)$, удовлетворяющих (2.2). Тогда некоторые два элемента множества $A$ линейно независимь над полем $\mathbb{Q}$.

ДокАзАТЕльство. Как уже отмечалось, многочлен $f_{n}(x)$ имеет степень $n-1$, является сепарабельным, причем все его корни вещественны и положительны. Разложим многочлен $f_{n}(x)$ на неприводимые множители над $\mathbb{Q}$. Если нашлась хотя бы одна неприводимая компонента, имеющая не менее двух корней из множества $A$, то результат следует из леммы 2. Поэтому без ограничения общности можно считать, что каждая неприводимая компонента имеет не более одного корня из множества $A$. Согласно лемме 1 , а также $(2.4)$ можно заключить, что если многочлен $f_{n}(x)$ имеет рациональные корни, то они необходимо целые. Заметим, что если хотя бы один элемент множества $A$ является целым числом, то либо найдутся два искомых линейно независимых над $\mathbb{Q}$ элемента множества $A$, либо справедливо включение $A \subset \mathbb{N}$. Но тогда и корень $v_{1} \in \mathbb{N}$. Однако корень $v_{1}$ должен являться решением уравнения $(2.2)$ при $k=1$. Если $n<16$, то это невозможно согласно результатам $\S 3$ работы [5]. Пусть теперь $n \geqslant 16$. Тогда справедлива следующая оценка (вычисленная с помощью системы компьютерной алгебры Maple V):

$$
\operatorname{arctg} \frac{\sqrt{1}}{1}+\operatorname{arctg} \frac{\sqrt{1}}{2}+\cdots+\operatorname{arctg} \frac{\sqrt{1}}{2 \cdot 16}>\pi .
$$

Оценка (2.6) вместе с равенством (2.2) при $k=1$ показывает, что $v_{1} \notin \mathbb{N}$. 
Итак, можно предполагать без ограничения общности, что каждая неприводимая компонента многочлена $f_{n}(x)$, имеющая корень из $A$, не является многочленом степени 1. Без ограничения общности число таких компонент равно $[n / 2]$. Но так как $n$ четно, то мы получим противоречие: степень многочлена $f_{n}(x)$ равна $n-1$, с другой стороны она не меньше $2[n / 2]=n$. Теорема доказана.

ЗАмЕчАНИЕ 1. Нетрудно обосновать оценку типа (2.6) для больших $n$ без применения компьютера.

В самом деле, функция $x \mapsto \operatorname{arctg} x$ выпукла вверх на отрезке $[0,1]$, поэтому для всех $x \in[0,1]$ справедливо неравенство $\operatorname{arctg} x \geqslant \pi x / 4$. Далее,

$$
\sum_{k=1}^{2 n} \operatorname{arctg} \frac{1}{k} \geqslant \frac{\pi}{4} \int_{1}^{2 n+1} \frac{d x}{x}=\frac{\pi}{4} \ln (2 n+1) .
$$

При $2 n+1>e^{4}$ требуемое неравенство выполняется.

2.2. Рассмотрим случай нечетного $n>3$. Следующая лемма представляет собой вариацию "постулата Бертрана".

Лемма 3. Пусть $n$ - нечетное натуральное число такое, что $n \geqslant 17$. Тогда интервал $((2 n+1) / 3, n)$ содержит хотя бъ одно простое число $p$, причем $p \nmid \sigma_{1,2 n}$.

ДокАзАТЕльство. Пусть сначала $n>32$. Пусть $r-$ натуральное число, удовлетворяющее свойствам

$$
\frac{n+r}{2}>\frac{2 n+1}{3}, \quad n>8+\frac{5 r}{3}, \quad n+r>48 .
$$

Такое число найдется, ибо при $n>32$ имеем $^{5}$

$$
\frac{n+2}{3}+1<\frac{3 n-8}{5}, \quad n+r>8+\frac{8 r}{3}>48 .
$$

Но тогда согласно [6; следствие 3.2$]$ интервал $((n+r) / 2, n-2)$ содержит хотя бы одно простое число $p$. В силу $(2.7)$ интервал $((2 n+1) / 3, n)$ также содержит $p$.

Пусть $n \in(16,32) \cap \mathbb{N}$, причем $n$ нечетно. Тогда интервал $((2 n+1) / 3, n)$ содержит простое число $p$, что проверяется непосредственно.

Покажем, что если $p \in((2 n+1) / 3, n)-$ простое число, то $p \nmid \sigma_{1,2 n}$. В самом деле, если $p \mid n$, то $n=p u$ для некоторого натурального $u \geqslant 2$. Но тогда

$$
\frac{2 p u}{3}<p<p u
$$

откуда $u<3 / 2$, что противоречит неравенству $u \geqslant 2$. Если же $p \mid(2 n+1)$, то $2 n+1=p v$ для некоторого натурального $v \geqslant 3$. В таком случае имеем

$$
\frac{2 n v}{3}<p v<n v \quad \Longrightarrow \quad\left\{\begin{array}{l}
2 n+1<n v, \\
2 n+1>\frac{2 n v}{3} .
\end{array}\right.
$$

Но тогда $v=3$, т.е. $p=(2 n+1) / 3$, что невозможно. Лемма доказана.

\footnotetext{
${ }^{5}$ Мы берем такое $r$, чтобы было выполнено неравенство $8+8 r / 3>48$.
} 
Напомним, что $A=\left\{v_{k}\right\}_{k=1}^{[n / 2]}$, где $v_{k}$ удовлетворяют $(2.3)$.

Лемма 4. Пусть $n>3$-нечетное натуральное число. Пусть далее $A \not \subset \mathbb{N}$. Тогда некоторье два элемента множества $A$ линейно независимы над полем $\mathbb{Q}$.

ДокАЗАТЕЛЬство. В силу [5; теорема 8] можно считать $n \geqslant 17$. Предположим, что каждая неприводимая над $\mathbb{Q}$ компонента многочлена $f_{n}(x)$ имеет не более одного корня из множества $A$ (в противном случае просто применим лемму 2 , по которой найдутся два линейно независимых над $\mathbb{Q}$ элемента множества $A$ ).

По условию $A \not \subset \mathbb{N}$. В таком случае без ограничения общности ни один элемент множества $A$ не является натуральным числом (иначе найдутся два элемента множества $A$ : натуральное число и число, не являющееся рациональным ${ }^{6}$; они будут линейно независимы над $\left.\mathbb{Q}\right)$. Это означает, что каждая неприводимая компонента многочлена $f_{n}(x)$, имеющая корень из множества $A$, не является многочленом степени 1 . Так как $|A|=(n-1) / 2$, а степень многочлена $f_{n}(x)$ равна $n-1$, то многочлен $f_{n}(x)$ необходимо разлагается над $\mathbb{Q}$ на неприводимые квадратичные множители в количестве $(n-1) / 2$. При этом каждый квадратичный множитель данного разложения имеет ровно один корень из множества $A$. Но тогда если никакие два элемента множества $A$ не являются линейно независимыми над $\mathbb{Q}$, то $\operatorname{Gal}_{\mathbb{Q}}\left(f_{n}\right) \cong Z_{2}$. Действительно, так как при указанных допущениях группа Галуа многочлена $f_{n}(x)$ над $\mathbb{Q}$ является элементарной абелевой 2-группой, то при $\left|\operatorname{Gal}_{\mathbb{Q}}\left(f_{n}\right)\right|>2$ непременно найдутся два неприводимых квадратичных множителя $u(x)$ и $v(x)$ такие, что их поля разложения линейно разделены над $\mathbb{Q}$. Однако тогда $\operatorname{Gal}_{\mathbb{Q}}(u v) \cong V_{4}$. Поэтому если $v_{j} \in A$ - корень $u(x)$, а $v_{l} \in A$ - корень $v(x)$, то найдется элемент $g \in \operatorname{Gal}_{\mathbb{Q}}(u v)$, действующий неподвижно на $v_{l}$ и нетривиально действующий на $v_{j}$. Но это означает, что $v_{j}$ и $v_{l}$ линейно независимы над $\mathbb{Q}$.

Итак, если $A \not \subset \mathbb{N}$, то либо найдутся два линейно независимых над $\mathbb{Q}$ элемента множества $A$, либо $\left|\operatorname{Gal}_{\mathbb{Q}}\left(f_{n}\right)\right|=2$. Пусть

$$
\widetilde{f}_{n}(x)=(-1)^{n-1} \sigma_{1,2 n}^{-1} f_{n}(x) .
$$

Тогда в силу леммы $1 \widetilde{f}_{n}(x) \in \mathbb{Z}[x]$, причем старший коэффициент равен единице. Так как по предположению $\left|\mathrm{Gal}_{\mathbb{Q}}\left(f_{n}\right)\right|=2$, то при допущении линейной зависимости над $\mathbb{Q}$ любых двух элементов множества $A$ справедливо следующее разложение на неприводимые квадратичные множители над $\mathbb{Q}$ :

$$
\tilde{f}_{n}(x)=\left(x^{2}+a x+b\right)\left(x^{2}+\alpha_{1} a x+\alpha_{1}^{2} b\right) \cdots\left(x^{2}+\alpha_{(n-3) / 2} a x+\alpha_{(n-3) / 2}^{2} b\right),
$$

где $a, b \in \mathbb{Z}$, причем $b>0, a<0$ (так как все корни многочлена $\widetilde{f}_{n}$ вещественны и положительны); числа $\alpha_{j}$ являются рациональными и положительными, причем $\alpha_{j} a \in \mathbb{Z}$, а также $\alpha_{j}^{2} b \in \mathbb{Z}$ для всех $j$.

\footnotetext{
${ }^{6}$ Напомним, что согласно лемме $1 \quad A \cap \mathbb{Q}=A \cap \mathbb{N}$.
} 
По лемме 3 найдется простое число $p$ из интервала $((2 n+1) / 3, n)$ такое, что $p \nmid \sigma_{1,2 n}$. Покажем, что $p \mid \sigma_{2 n-1,2 n}$, но $p^{2} \nmid \sigma_{2 n-1,2 n}$. В самом деле, $2 p<2 n$, a $3 p>2 n$. Поэтому $p \mid \sigma_{2 n-1,2 n}\left(\sigma_{2 n-1,2 n}\right.$ представляет собой сумму произведений натуральных чисел, не превосходящих $2 n$, причем в каждом таком произведении вычеркивается ровно одно натуральное число, меньшее $2 n+1$ ). Далее $p^{2} \nmid \sigma_{2 n-1,2 n}$. Действительно, все слагаемые в $\sigma_{2 n-1,2 n}$ делятся на $p^{2}$, кроме двух: одно получается из набора $[1,2 n] \cap \mathbb{N}$ вычеркиванием $p$, а другое вычеркиванием $2 p$. После сокращения на $p$ в этих двух слагаемых их сумма не будет делиться на $p$, так как полученная сумма есть утроенное произведение натуральных чисел из набора $[1,2 n] \cap(\mathbb{N} \backslash\{p, 2 p\})$.

Поскольку $p \nmid \sigma_{1,2 n}$, то $p \mid \widetilde{f}_{n}(0)$, но $p^{2} \nmid \widetilde{f}_{n}(0)$. Таким образом,

$$
\nu\left(\widetilde{f}_{n}(0)\right)=1 .
$$

Но тогда из разложения (2.8) мы получаем следующее равенство:

$$
1=\nu\left(\tilde{f}_{n}(0)\right)=\nu(b)+\sum_{i=1}^{(n-3) / 2}\left(\nu(b)+2 \nu\left(\alpha_{i}\right)\right),
$$

где все слагаемые суммы справа суть целые неотрицательные числа ${ }^{7}$. Если $\nu(b)=1$, то значение суммы справа не меньше $1+(n-3) / 2$, что в силу $(2.9)$ возможно лишь при $n \leqslant 3$. Однако по условию $n>3$. Противоречие. Если же $\nu(b)=0$, то равенство (2.9) выполняется лишь тогда, когда для некоторого $i_{0} \in[1,(n-3) / 2] \cap \mathbb{N}$ верно $2 \nu\left(\alpha_{i_{0}}\right)=1$, а для всех индексов $i \neq i_{0}$ - соответственно $2 \nu\left(\alpha_{i}\right)=0$. Но это означает справедливость равенства $\nu\left(\alpha_{i_{0}}\right)=1 / 2$, что невозможно в силу рациональности числа $\alpha_{i_{0}}$. Снова получаем противоречие. Лемма доказана.

Разберем случай $A \subset \mathbb{N}$. В этом случае корень $v_{1} \in A$ уравнения $(2.3)$ при $k=1$ также является натуральным числом. Справедлива следующая оценка (вычисленная с помощью системы компьютерной алгебры Maple V):

$$
\operatorname{arctg} \frac{\sqrt{1}}{1}+\operatorname{arctg} \frac{\sqrt{1}}{2}+\cdots+\operatorname{arctg} \frac{\sqrt{1}}{2 \cdot 199}>2 \pi .
$$

Оценка (2.10) вместе с равенством (2.3) при $k=1$ показывает, что $v_{1} \notin \mathbb{N}$ при всех нечетных $n \geqslant 199$.

Теорема 2. Пусть $n$ - нечетное натуральное число, большее 3 , а многочлен $f_{n}(x)$ определен согласно (2.4). Пусть $A=\left\{v_{k}\right\}_{k=1}^{n / 2}$ - множество корней многочлена $f_{n}(x)$, удовлетворяющих (2.3). Тогда некоторые два элемента множества А линейно независимы над полем $\mathbb{Q}$.

ДокАЗАТЕЛЬСтво. В силу леммы 4, оценки (2.10), а также теоремы 8 работы [5] достаточно доказать, что для всех нечетных $n \in[17,197] \cap \mathbb{N}$ уравнение (2.3) при $k=1$ неразрешимо в натуральных числах. Дальнейшие оценки вычислены с помощью Maple V.

\footnotetext{
${ }^{7}$ То есть $\nu(b) \in \mathbb{Z}_{\geqslant 0}$, а также $2 \nu\left(\alpha_{i}\right) \in \mathbb{Z}_{\geqslant 0}$ для всех $i$.
} 
Заметим, что

$$
\operatorname{arctg} \frac{\sqrt{4}}{1}+\cdots+\operatorname{arctg} \frac{\sqrt{4}}{2 \cdot 17}>2 \pi .
$$

Поэтому при нечетных $n \geqslant 17$ если корень $v_{1}$ уравнения $(2.10)$ при $k=1$ является натуральным числом, то $v_{1} \in\{1,2,3\}$. При этом если $n \geqslant 37$, то $v_{1}=1$, ибо справедлива оценка

$$
\operatorname{arctg} \frac{\sqrt{2}}{1}+\cdots+\operatorname{arctg} \frac{\sqrt{2}}{2 \cdot 37}>2 \pi
$$

С другой стороны, решение уравнения

$$
\operatorname{arctg} \frac{\sqrt{x}}{1}+\cdots+\operatorname{arctg} \frac{\sqrt{x}}{2 \cdot m}=2 \pi
$$

больше 1 при $m=197$. Поскольку с ростом $m$ корень $v_{1}$ уравнения $(2.13)$ уменьшается, то при $n \in[17,197] \cap \mathbb{N}$ заведомо $v_{1} \neq 1$. Итак, из $(2.11)$ и $(2.12)$ вытекает, что $v_{1} \in\{2,3\}$, причем $n<37$. Решение уравнения $(2.13)$ при $m=35$ больше 2 , при $m=19$ меньше 3 , а при $m=17$ больше 3 . Это означает (так как с ростом $m$ корень уравнения (2.13) уменьшается), что уравнение (2.13) не имеет целочисленных, а значит (в силу леммы 1), и рациональных решений для всех нечетных $n>3$. Теорема доказана.

СледСтвиЕ 1. В задаче оптимального управления (1.1) при всех натуральных $n>3$ можно подобрать оператор $C$ так, чтобъ (1.1) имела оптимальное решение, управление которого за конечное время проходит всюду плотную обмотку 2-мерного клиффордова тора.

ДокАЗАТЕльство. Для доказательства достаточно воспользоваться теоремами 1 и 2 настоящей работы, а также результатом [5; теорема 3].

\section{Список литературы}

[1] М.И. Зеликин, Л.В. Локуциевский, Р. Хильдебранд, "Геометрия окрестностей особых экстремалей в задачах с многомерным управлением", Математическая теория управления и дифференциальные уравнения, Сб. статей, Тр. МИАН, 277, МАИК, М., 2012, 74-90; англ. пер.: M. I. Zelikin, L. V. Lokutsievskiy, R. Hildebrand, "Geometry of neighborhoods of singular trajectories in problems with multidimensional control", Proc. Steklov Inst. Math., 277 (2012), 67-83.

[2] I. Kupka, "Generic properties of extremals in optimal control problems", Differential geometric control theory (Houghton, Mich., 1982), Progr. Math., 27, Birkhäuser, Boston, MA, 1983, 310-315.

[3] M. I. Zelikin, V.F. Borisov, Theory of chattering control with applications to astronautics, robotics, economics, and engineering, Systems Control Found. Appl., Birkhäuser, Boston, MA, 1994, xvi+242 pp. 
[4] А. А. Милютин, А. Е. Илютович, Н. П. Осмоловский, С. В. Чуканов, Оптимальное управление в линейных системах, Наука, М., 1993, 269 с.

[5] М.И. Зеликин, Д. Д. Киселев, Л.В. Локуциевский, "Оптимальное управление и теория Галуа", Матем. сб., 204:11 (2013), 83-98; англ. пер.: M. I. Zelikin, D. D. Kiselev, L. V. Lokutsievskii, "Optimal control and Galois theory", Sb. Math., 204:11 (2013), 1624-1638.

[6] F. Hajir, "Algebraic properties of a family of generalized Laguerre polynomials", Canad. J. Math., 61:3 (2009), 583-603.

\section{Денис Дмитриевич Киселев}

(Denis D. Kiselev)

Поступила в редакцию

Всероссийская академия внешней торговли, 09.01 .2015 и 09.10 .2015

г. Москва

E-mail: denmexmath@yandex.ru 